Research Paper

\title{
Bullous Pemphigoid and Diabetes medications: A disproportionality analysis based on the FDA Adverse Event Reporting System
}

\author{
Liting Huang1,2\#, Ying Liu", Huijun Li1,2, Weicun Huang1,2, Ruirui Geng ${ }^{1,2}$, Zaixiang Tang1,2® and Yiguo \\ Jiang $4^{\bowtie}$ \\ 1. Department of Biostatistics, School of Public Health, Medical College of Soochow University, Suzhou, 215123, China. \\ 2. Jiangsu Key Laboratory of Preventive and Translational Medicine for Geriatric Diseases, Medical College of Soochow University, Suzhou, 215123, China. \\ 3. National Institute for Food and Drug Control, Beijing, 102629, China. \\ 4. Department of pharmacy, The Affiliated Suzhou Science \& Technology Town Hospital of Nanjing Medical University, Suzhou, 215153, China. \\ \#These authors contributed equally to this work.
}

$\square$ Corresponding authors: Yiguo Jiang, TEL: 0512-33322120; E-mail: jiangyiguo0515@126.com; Department of Pharmacy, The Affiliated Suzhou Science \& Technology Town Hospital of Nanjing Medical University, Suzhou, 215153, China. Zaixiang Tang, TEL: 0512-65883227; E-mail: tangzx@suda.edu.cn; Department of Biostatistics, School of Public Health, Medical College of Soochow University, Suzhou, 215123, China.

(C) The author(s). This is an open access article distributed under the terms of the Creative Commons Attribution License (https://creativecommons.org/licenses/by/4.0/). See http://ivyspring.com/terms for full terms and conditions.

Received: 2020.11.03; Accepted: 2021.02.15; Published: 2021.03.03

\begin{abstract}
Background: The world's first Diabetes Medications (Insulin) was marketed in October 1923. Some studies suggested the association of diabetes medications with Bullous Pemphigoid (BP), especially the Dipeptidyl Peptidase 4 (DPP-4) inhibitors. The study aims to detect an association between diabetes medications (focusing on DPP-4 inhibitors) and bullous pemphigoid based on FDA Adverse Event Reporting System (FAERS).

Methods: All spontaneous reports of diabetes medications inhibitors-related BP recorded in the FAERS between March 2004 and August 2020 were included in the present study. Disproportionality analysis was performed to find the signal between diabetes medications and BP. The Chi-Squared with Yates' correction $\left(X^{2} Y\right.$ ates $)$, proportional reporting ratio (PRR) and the lower limit of the $95 \%$ confidence interval of the Reporting Odds Ratio (ROR 025 ) were calculated as a measure. A signal was detected when ROR $\mathrm{R}_{025}$ $>1, P R R>2, X^{2}$ Yates $>4$ and at least 3 cases.

Results: There were 3770 reports for BP in FAERS. The strongest signal for diabetes medications-BP association were DDP-4 inhibitors (ROR 025 : 13.700, PRR: 15.408), followed by Meglitinides (ROR 025 : 12.708, PRR: 16.777), Non-sulfonylureas (ROR025: 6.434, PRR: 7.016), Alpha-glucosidase inhibitors (ROR 025 : 6.105, PRR: 10.738), Sulfonylureas (ROR $025: 2.655$, PRR: 3.200).

Conclusions: This study detected a strong signal between BP and DDP-4 inhibitors, alpha-glucosidase inhibitors, meglitinides, non-sulfonylureas, and sulfonylureas in FAERS. The signal was significantly higher with alogliptin than with the other DPP-4 inhibitors. The study doesn't suggest the association between the incretin mimetics, insulin, SGLT-2 inhibitors, thiazolidinediones and BP in FAERS.
\end{abstract}

Key words: diabetes medications; dipeptidyl peptidase 4 inhibitors; Bullous Pemphigoid; FAERS; drug safety

\section{Introduction}

Bullous pemphigoid (BP) is a rare acquired autoimmune skin condition. It usually develops on areas of skin that often flex, such as the lower abdomen, upper thighs, or armpits. The clinical manifestations of BP include tense bullae, urticarial skin lesions and pruritus, oral mucous membrane erosions that may be present in $10-20 \%$ of patients [1-3]. In some patients, eczema-like erythema may proceed for months or even for many years as a prodromal phase before BP develops [1]. A retrospective monocentric cohort study confirmed that BP was associated with high mortality [4]. BP is 
most common in older adults, the incidence of BP appears to be equal in men and women and no known ethnic or racial predilection is detected for developing bullous pemphigoid [5]. BP is caused by an autoimmune reaction against bullous pemphigoid antigen 180 (BP180) and/or bullous pemphigoid antigen 230 (BP230), both BP180 and BP230 are a major structural component of hemidesmosomes [6, 7]. BP230 localizes intracellularly and associates with the hemidesmosomal plaque, BP180 is a transmembrane glycoprotein with an extracellular domain [6]. Antibodies against both BP180 and BP230, as measured by ELISA, are used for the diagnosis of bullous pemphigoid [3]. But the exact reason for this abnormal immune response is unknown, although it sometimes can be triggered by taking certain medications, trauma, burns, radiotherapy, ultraviolet irradiation, the phenomenon of epitope spreading or genetic factor [8, 9]. There are more than 50 medications have been associated with BP development [10].

The world's first Diabetes Medications (Insulin) was marketed in October 1923. A study suggested the association of diabetes mellitus with BP [11]. Meanwhile, DPP-4 inhibitors (also known as "gliptins") and tolbutamide were associated with BP in the literature [10]. So, it was necessary to analyze the association between diabetes medications and BP. For individual diabetes medications, nonsulfonylureas (including the metformin) and DPP-4 inhibitors should be focused on. Metformin was a classic antihyperglycemic drug and the top treatment choice for type 2 diabetes. Metformin always was used in combination with DPP-4 inhibitors. DPP-4 inhibitors are a class of diabetes medications that are used with diet and exercise to control high blood sugar in adults with type 2 diabetes. DPP- 4 inhibitors lower blood sugar by helping the body increase the level of the hormone insulin after meals. Insulin helps move sugar from the blood into the tissues, so the body can use the sugar to produce energy and keep blood sugar levels stable. The DPP-4 inhibitors may induce anti-basement membrane zone antibodies or other structurally close antibodies [12], leading to BP. Inhibition of DPP-4 has been shown to enhance the recruitment of eosinophils into the dermis, which may contribute to the blister formation and tissue damage observed in BP [13]. The inhibition of gliptins may cause the activation of eosinophils by a CCL11/ eotaxin-mediated mechanism. The activation of eosinophils and lymphocyte infiltration substantially contributes to the appearance of blisters and tissue damage in bullous pemphigoid. On the other hand, DPP-4 inhibitors may alter the antigenic properties of the epidermal basement membrane [14]. Even though an increasing number of cases of $\mathrm{BP}$ induced by DPP-4 inhibitor was reported in the literature, the exact mechanism underlying this association remains unclear and needs to be elucidated [8].

Previously, some case reports supported the hypothesis that there is a risk of BP in patients exposed to DPP-4 inhibitors [12, 14-20]. Some retrospective studies suggested that the use of DPP-4 inhibitors is associated with the development of BP in patients with diabetes [21, 22]. A meta-analysis suggested that DPP-4 inhibitor exposure is associated with a significantly increased risk for BP [23]. And the warnings and precautions of DPP-4 inhibitors' latest label in the FDA showed that there have been reports of bullous pemphigoid requiring hospitalization. But other types of diabetes medications' labels in the FDA didn't include the warnings about bullous pemphigoid.

Data mining algorithms (DMAs) are currently and routinely used by pharmacovigilance experts for quantitative signal detection [24]. The accuracy of data mining techniques has been already tested retrospectively to determine if already known safety issues would have been detected 'earlier' [25]. Some scholars conducted disproportionality analyses based on DMAs for all spontaneous reports from the French, European, Japanese, WHO and Spanish Pharmacovigilance Database [8, 9, 26-28]. These studies based on the pharmacovigilance databases all showed a significant association between DPP-4 inhibitors and BP.

FDA Adverse Event Reporting System (FAERS) was the pharmacovigilance database of the United States. We investigated the association between all types of diabetes medications (focused on DPP-4 inhibitors) and BP using the data from FAERS based on DMAs in this study. In addition, the pooled analysis based on DMAs between the DPP-4 inhibitors and BP was made by combining French, American, Japanese, WHO and Spanish Pharmacovigilance Database in the study.

\section{Materials and Methods}

\section{Study Design}

A retrospective analysis was conducted to comparatively assess BP reports with Diabetes Medications. Acetaminophen was considered as a negative control, whereas furosemide illustrated descriptive positive control $[9,10]$.

\section{Data source}

Data in the present study were obtained from the public release of the OpenVigil FDA (https://openvigil.pharmacology.uni-kiel.de/openvi gilfda.php), which covers the period from March 2004 
through August 2020 in the FAERS.

The data currently used in OpenVigil FDA was obtained from FAERS [29, 30]. OpenVigil FDA is a pharmacovigilance tool to extract and analyze FAERS data using the OpenFDA API for accessing the FDA drug-event-database with the additional OpenFDA drug mapping and duplicate detection functionality, OpenFDA aims at providing clean and curated access to the underlying AERS and can count reports stratified to an extraction condition [29], and it overcame some disadvantages of FAERS.

In the study, DPP-4 inhibitors were limited to the approved drugs by the FDA (sitagliptin, saxagliptin, linagliptin, alogliptin). The study analyzed the pooled DPP-4 inhibitors and each DPP-4 inhibitor individually. For reducing the interference from gender, this study also analyzed the pooled DPP-4 inhibitors and each DPP-4 inhibitor individually by a different gender.

Most patients with DDP-4 inhibitors received combinations of other medications. A sensitivity analysis was made after excluding cases where drugs other than DPP-4 inhibitors were suspected in the BP occurrence (Supplementary Table 1) to reduce the confounding bias.

Diabetes medications other than DPP-4 inhibitors analyzed in the study were listed in Supplementary Table 2 . The study also analyzed the association between diabetes medications and BP after excluding the cases of combined use of DPP-4 inhibitors to reduce the DDP-4 inhibitors' interference.

\section{Definition of adverse events}

Adverse events in the OpenVigil FDA were coded according to the terminology preferred by the Medical Dictionary for Regulatory Activities (MedDRA) Preferred Terms (PTs). For the disproportionality analysis, pemphigoid (PT10034277) were selected for mining according to the MedDRA 22.0.

\section{Data mining algorithms}

Data mining algorithms (DMAs) can be classified in the frequentist and Bayesian approach. The frequentist methods are based on the same principles of calculation using the $2 \times 2$ table (Supplementary Table 3) [31]. The study calculated proportional reporting ratio (PRR), Reporting Odds Ratio (ROR), ROR 025, and Chi-Squared with Yates' correction $\left(X^{2}\right.$ Yates $)$ based on the frequentist approach from adverse drug reaction reports determining whether the combination of drug and adverse event are related.

These values were calculated on the Open
Vigil-2×2 contingency table calculator (https:// openvigil.pharmacology.uni-kiel.de/contingencytable-calculator.php) in the study.

For the study, when PRR $>2, \chi^{2}$ Yates $>4(=p<$ $0.05)$, the lower limit of the $95 \%$ confidence interval of the $\mathrm{ROR}\left(\mathrm{ROR}_{025}\right)$ is greater than one and at least 3 cases as minimal criteria for a signal of disproportionality [31, 32].

\section{Results}

\section{Case selection}

During the study period (between 2004 and 2020), 12254196 adverse drug reaction reports were entered in the OpenVigil FDA. Among these, 89277 adverse drug reaction reports were related to DPP-4 inhibitors, and 3770 adverse drug reaction reports were related to BP. Among these DPP-4 inhibitors' reports, 383 reports were related to BP (alogliptin, $\mathrm{n}=$ 70 ; linagliptin, $\mathrm{n}=51$; sitagliptin, $\mathrm{n}=250$; saxagliptin, $\mathrm{n}=17), 5$ of them involved two or more DPP-4 inhibitors.

\section{Characteristics of the DDP-4 inhibitors and control group}

For the gender, the reaction tended to be more common in male $(50.91 \%, 61.43 \%, 66.67 \%, 44.40 \%$ and $35.29 \%$ of pooled DPP-4 inhibitors-, alogliptin-, linagliptin-, sitagliptin-, saxagliptin-related cases, respectively) and elderly people-at least 75 years $(52.22 \%, 68.57 \%, 35.29 \%, 49.20 \%$ and $70.59 \%$ of pooled DPP-4 inhibitors-, alogliptin-, linagliptin-, sitagliptin-, saxagliptin-related cases, respectively). For the control group, the gender distribution is different, acetaminophen-related cases tended to be more common in female $(58.33 \%)$, but furosemide-related cases tended to be more common in male $(52.58 \%)$. The entire control group tended to be elderly people-at least 75 years $(45.00 \%$ and $55.32 \%$ of acetaminophen- and furosemide-related cases, respectively). The age distribution of these cases was similar to the general BP population, but the gender distribution was different from the general BP population [5]. The characteristics of DDP-4 inhibitors and the control group were summarized in Table 1.

\section{BP and DDP-4 inhibitors in the FAERS}

The study made a general disproportionality analysis between DDP-4 inhibitors and BP in the FAERS. BP cases were reported more frequently for DPP-4 inhibitors than for the control group. For the DMAs result between pooled DDP-4 inhibitors and $\mathrm{BP}$, it showed a signal, with the $\mathrm{ROR}_{025}$, PRR, the number of adverse events and $\chi^{2}$ Yates of 13.916, 15.408, 383, and 4624.373, respectively. For the DMAs result 
between the furosemide and BP, it showed a signal, with the ROR 025 , PRR, the number of adverse events and $X^{2}$ Yates of 3.838, 4.294, 329, and 756.041, respectively. For the DMAs result between the acetaminophen and BP, it didn't show a signal, with the $\mathrm{ROR}_{025}$, PRR, the number of adverse events and $X^{2}$ Yates of $0.418,0.540,60$, and 22.657, respectively. For the DMAs result between each DDP-4 inhibitor and $\mathrm{BP}$, it showed a signal. The largest disproportionality corresponded to alogliptin, followed in decreasing order by linagliptin, sitagliptin, and saxagliptin. The DMAs result between pooled DDP-4 inhibitors and $\mathrm{BP}$ in the gender did not suggest the different disproportionality result between the male and female. The results were summarized in Table 2.

The study also made a sensitivity disproportionality analysis between DDP-4 inhibitors and $\mathrm{BP}$ in the FAERS. For the DMAs result between pooled DDP-4 inhibitors and BP, it showed a signal, with the $\mathrm{ROR}_{025}$, PRR, the number of adverse events and $X^{2}$ Yates of 13.700, 15.362, 298, and 3672.735, respectively. The analysis values were different from the general disproportionality analysis, but it also displayed high disproportionality regarding the association between pooled DDP-4 inhibitors and BP. For individual DPP-4 inhibitors, the disproportionality order was the same as in the general disproportionality analysis. The results were summarized in Table 2.

\section{BP and DDP-4 inhibitors in the Pooled databases}

By combining the results of the study with those previous studies conducted over the FPVD (France), JADER (Japan), FEDRA (Spanish) and VigiBase (WHO) databases [8, 9, 26, 27]. For the DMAs result between DDP-4 inhibitors and BP in the Pooled databases, it showed a signal, with the $\mathrm{ROR}_{025}$, PRR, the number of adverse events and $\chi^{2}$ Yates of 60.276 , $62.711,1932$, and 87122.550, respectively (Table 3).

\section{BP and other diabetes medications in the FAERS}

For the DMAs result between the nonsulfonylureas and BP, it showed a signal, with the $R_{02}$, PRR, the number of adverse events and $\chi^{2}$ Yates of $6.434,7.016,584$, and 2541.646, respectively. After excluding case subjects who received DPP-4 inhibitors to reduce the interference of DPP-4 inhibitors, significant disproportionality did not disappear for case subjects receiving the non-sulfonylureas. For the DMAs result between the other individual diabetes medications and BP, the alpha-glucosidase inhibitors, meglitinides and sulfonylureas showed disproportionality regardless of whether excluding case subjects who received DPP-4 inhibitors, but the incretin mimetics (also known as GLP-1 Agonists), insulin, SGLT-2 inhibitors and thiazolidinediones did not show disproportionality regardless of whether excluding case subjects who received DPP-4 inhibitors. The OpenVigil FDA did not receive the report between amylin analogs and BP. These results were summarized in Table 4.

\section{Discussion}

The DPP-4 inhibitors-related BP cases tended to be more common in males (presumably because DPP-4 inhibitors were used more often in males than in females [27]) and elderly people (at least 75 years). The effect of DPP-4 inhibitors on BP did not have a statistical difference in gender in the FAERS. It was different from the result of a hospital-based Swiss-French study and a Finnish nationwide registry study, which found that the effect of DPP-4 inhibitors on $\mathrm{BP}$ had a statistical difference in gender $[33,34]$.

These results showed disproportionality for BP and DPP-4 inhibitors in the entire pharmacological databases and the FAERS regardless of whether excluding cases where drugs other than DPP-4 inhibitors were suspected in the BP occurrence, which was consistent with those reported in previous studies conducted in other countries' pharmacovigilance databases [8, 9, 26-28]. Analysis of each DPP-4 inhibitor separately also showed a significant association. Alogliptin showed higher $\mathrm{ROR}_{025}$ than other DPP-4 inhibitors, followed in decreasing order by linagliptin, sitagliptin and saxagliptin. It was different from the previous studies $[8,9,26-28]$, presumably because the different regulatory Agencies approved the different DPP-4 inhibitors. For example, the FDA did not approve the vildagliptin, which appeared a higher risk than the others in other countries' pharmacovigilance databases' study [9, 26-28]. It was interesting to specify that sitagliptin was the most prescribed DPP-4 inhibitor in the USA [35]. However, disproportionality analyses confirmed a higher risk in alogliptin. No clear reason has been found to explain the higher association of alogliptin with the development of BP compared with the other DPP-4 inhibitors. For negative control (acetaminophen), the study did not show disproportionality. For the positive control (furosemide), the study showed disproportionality. The results of the control group were consistent with those reported in previous studies $[9,10]$. 
Table 1. General characteristics of cases of bullous pemphigoid associated with DDP-4 inhibitors and the control group in FAERS

\begin{tabular}{|c|c|c|c|c|c|c|c|}
\hline & Gliptins & Alogliptin & Linagliptin & Sitagliptin & Saxagliptin & Acetaminophen & Furosemide \\
\hline \multicolumn{8}{|l|}{ Gender } \\
\hline Female & $154(40.21 \%)$ & $21(30.00 \%)$ & $12(23.53 \%)$ & $117(46.80 \%)$ & $10(58.82 \%)$ & $35(58.33 \%)$ & $135(41.03 \%)$ \\
\hline Male & $195(50.91 \%)$ & $43(61.43 \%)$ & $34(66.67 \%)$ & $111(44.40 \%)$ & $6(35.29 \%)$ & $23(38.33 \%)$ & $173(52.58 \%)$ \\
\hline UK & $34(8.88 \%)$ & $6(8.57 \%)$ & $5(9.80 \%)$ & $22(8.80 \%)$ & $1(5.88 \%)$ & $2(3.33 \%)$ & $1(0.30 \%)$ \\
\hline \multicolumn{8}{|l|}{ Age } \\
\hline$\leq 44$ & $7(1.83 \%)$ & $2(2.86 \%)$ & $3(5.88 \%)$ & $2(0.80 \%)$ & $0(0.00 \%)$ & $2(3.33 \%)$ & $1(0.30 \%)$ \\
\hline $45-64$ & $33(8.62 \%)$ & $6(8.57 \%)$ & $4(7.84 \%)$ & $24(9.60 \%)$ & $1(5.88 \%)$ & $13(21.67 \%)$ & $31(9.42 \%)$ \\
\hline $65-74$ & $82(21.41 \%)$ & $7(10.00 \%)$ & $15(29.41 \%)$ & $60(24.00 \%)$ & $1(5.88 \%)$ & $12(20.00 \%)$ & $80(24.32 \%)$ \\
\hline$\geq 75$ & $200(52.22 \%)$ & $48(68.57 \%)$ & $18(35.29 \%)$ & $123(49.20 \%)$ & $12(70.59 \%)$ & $27(45.00 \%)$ & $182(55.32 \%)$ \\
\hline UK & $61(15.93 \%)$ & $7(10.00 \%)$ & $11(21.57 \%)$ & $41(16.40 \%)$ & $3(17.65 \%)$ & $6(10.00 \%)$ & $35(10.64 \%)$ \\
\hline Total & 383 & 70 & 51 & 250 & 17 & 60 & 329 \\
\hline
\end{tabular}

Table 2. The general and sensitivity DMAs results between DDP-4 inhibitors/control group and bullous pemphigoid

\begin{tabular}{|c|c|c|c|c|c|}
\hline Drugs & a & $\chi^{2}$ Yates & PRR & ROR & $\mathrm{ROR}_{025}$ \\
\hline Gliptins & 383 & 4624.373 & 15.408 & 15.470 & 13.916 \\
\hline Male & 195 & 1830.141 & 12.454 & 12.511 & 10.783 \\
\hline Female & 154 & 2094.144 & 17.102 & 17.161 & 14.529 \\
\hline Sensitivity analysis result & 298 & 3672.735 & 15.362 & 15.425 & 13.700 \\
\hline Alogliptin & 70 & 6065.722 & 91.544 & 94.118 & 74.054 \\
\hline Male & 43 & 3069.234 & 76.767 & 79.363 & 58.359 \\
\hline Female & 21 & 1707.185 & 88.469 & 90.300 & 58.449 \\
\hline Sensitivity analysis result & 58 & 5396.474 & 98.125 & 101.101 & 77.701 \\
\hline Linagliptin & 51 & 649.065 & 15.107 & 15.172 & 11.501 \\
\hline Male & 34 & 460.425 & 16.168 & 16.276 & 11.580 \\
\hline Female & 12 & 82.490 & 9.513 & 9.532 & 5.398 \\
\hline Sensitivity analysis result & 39 & 526.123 & 15.945 & 16.018 & 11.675 \\
\hline Sitagliptin & 250 & 2535.917 & 12.830 & 12.874 & 11.322 \\
\hline Male & 111 & 733.032 & 8.979 & 9.009 & 7.434 \\
\hline Female & 117 & 1593.627 & 16.776 & 16.834 & 13.941 \\
\hline Sensitivity analysis result & 191 & 1923.614 & 12.587 & 12.630 & 10.916 \\
\hline Saxagliptin & 17 & 68.786 & 6.203 & 6.213 & 3.856 \\
\hline Male & 6 & 8.287 & 3.489 & 3.493 & 1.566 \\
\hline Female & 10 & 74.318 & 10.283 & 10.305 & 5.530 \\
\hline Sensitivity analysis result & 14 & 61.864 & 6.682 & 6.694 & 3.958 \\
\hline Acetaminophen & 60 & 22.657 & 0.540 & 0.540 & 0.418 \\
\hline Male & 35 & 3.018 & 0.735 & 0.735 & 0.526 \\
\hline Female & 23 & 12.803 & 0.473 & 0.473 & 0.313 \\
\hline Without Gliptins & 59 & 22.546 & 0.538 & 0.538 & 0.416 \\
\hline Furosemide & 329 & 756.041 & 4.294 & 4.298 & 3.838 \\
\hline Male & 135 & 148.620 & 2.849 & 2.852 & 2.393 \\
\hline Female & 173 & 597.785 & 5.730 & 5.736 & 4.898 \\
\hline Without Gliptins & 317 & 717.700 & 4.246 & 4.250 & 3.788 \\
\hline
\end{tabular}

$\chi^{2}$ Yates: The Chi-Squared with Yates' correction.

ROR $_{025}$ : The lower limit of the $95 \%$ confidence interval of the ROR.

Sensitivity analysis result: Excluding cases where drugs other than DDP-4 inhibitors were suspected in the BP occurrence.

a: The number of adverse events corresponding to the drug.

Table 3. DMAs result of data from five pharmacovigilance databases: FPVD, JADER, FEDRA, VigiBase, and FAERS

\begin{tabular}{llllll}
\hline Database & $\mathrm{a}$ & $\chi^{2}$ Yates & PRR & ROR & ROR $_{025}$ \\
\hline FPVD & 42 & 1867.135 & 65.380 & 67.535 & 47.062 \\
JADER & 392 & 9163.440 & 84.988 & 87.558 & 72.608 \\
FEDRA & 45 & 1627.011 & 69.770 & 71.355 & 47.921 \\
VigiBase & 1070 & 118159.250 & 175.504 & 179.430 & 166.362 \\
FAERS & 383 & 4624.373 & 15.408 & 15.470 & 13.916 \\
Total & 1932 & 87122.550 & 62.711 & 63.493 & 60.276 \\
\hline
\end{tabular}

$\chi^{2}$ Yates: The Chi-Squared with Yates' correction.

$\mathrm{ROR}_{025}$ : The lower limit of the $95 \%$ confidence interval of the ROR.

a: The number of adverse events corresponding to the drug.
Table 4. Association between antihyperglycemic drug exposure and bullous pemphigoid occurrence measured by disproportionality analysis

\begin{tabular}{llllll}
\hline Drugs & $\mathrm{a}$ & $\chi^{2}$ Yates & PRR & ROR & $\mathrm{ROR}_{025}$ \\
\hline Alpha-glucosidase inhibitors & 12 & 96.178 & 10.738 & 10.770 & 6.105 \\
Without Gliptins & 11 & 91.438 & 11.144 & 11.179 & 6.179 \\
Amylin analogs & 0 & $\mathrm{NA}^{*}$ & $\mathrm{NA}^{*}$ & $\mathrm{NA}^{*}$ & $\mathrm{NA}^{*}$ \\
Without Gliptins & 0 & $\mathrm{NA}^{*}$ & $\mathrm{NA}^{*}$ & $\mathrm{NA}^{*}$ & $\mathrm{NA}^{*}$ \\
Incretin mimetics & 34 & 3.593 & 0.714 & 0.714 & 0.509 \\
Without Gliptins & 31 & 4.555 & 0.673 & 0.673 & 0.473 \\
Insulin & 142 & 33.700 & 1.642 & 1.643 & 1.389 \\
Without Gliptins & 117 & 12.844 & 1.405 & 1.405 & 1.169 \\
Meglitinides & 49 & 702.257 & 16.777 & 16.858 & 12.708 \\
Without Gliptins & 41 & 549.259 & 15.836 & 15.908 & 11.685 \\
Non-sulfonylureas & 584 & 2541.646 & 7.016 & 7.028 & 6.434 \\
Without Gliptins & 429 & 1462.319 & 5.680 & 5.688 & 5.143 \\
SGLT-2 inhibitors & 27 & 5.401 & 1.597 & 1.597 & 1.094 \\
Without Gliptins & 22 & 2.716 & 1.459 & 1.459 & 0.959 \\
Sulfonylureas & 113 & 163.667 & 3.200 & 3.202 & 2.655 \\
Without Gliptins & 82 & 79.936 & 2.637 & 2.638 & 2.120 \\
Thiazolidinediones & 51 & 1.728 & 1.216 & 1.217 & 0.923 \\
Without Gliptins & 32 & 1.571 & 0.790 & 0.790 & 0.558
\end{tabular}

$\chi^{2}$ Yates: The Chi-Squared with Yates' correction.

$\mathrm{ROR}_{025}$ : The lower limit of the $95 \%$ confidence interval of the ROR.

a: The number of adverse events corresponding to the drug.

NA: Not applicable due to the low number of case reports $(a<3)$.

The alpha-glucosidase inhibitors, meglitinides, non-sulfonylureas and sulfonylureas with BP showed disproportionality regardless of whether excluding case subjects who received DPP-4 inhibitors. It was different from the results of the JADER database and Finnish nationwide case-control study at Rambam Health Care Campus, Haifa, Israel [27, 36]. The incretin mimetics, insulin, SGLT-2 inhibitors and thiazolidinediones with BP did not show disproportionality regardless of whether excluding case subjects who received DPP-4 inhibitors. The above results were different from the results of the JADER database, which showed that the significant ROR disappeared for case subjects receiving the other individual diabetes medications after excluding case subjects who received DPP-4 inhibitors [27]. For the alpha-glucosidase inhibitors, meglitinides, nonsulfonylureas, and sulfonylureas, perhaps because the FAERS database had more reports than the JADER database or the association of diabetes mellitus with 
BP. In those early reports, the association of diabetes mellitus with BP had been analyzed, and possible underlying mechanisms that increased skin fragility due to elevated glucose levels and the induction of autoantibody production by glycosylation of dermal proteins were suggested [11]. The DMAs results between other individual diabetes medications and BP did not change after excluding case subjects who received DPP-4 inhibitors. It meant that maybe other types of diabetes medications did not interact with DPP-4 inhibitors on BP.

Our study has limitations. The FAERS database was a spontaneous reporting system rather than a mandatory reporting system, the reporters consisted of patients, caregivers, and manufacturers. FDA did not receive reports for every adverse event or medication error that occurs with a product. This introduced an inevitable selection bias, and reporting biases may be differential across different drugs. There was no specific role to check the data in the report, the entry errors couldn't be controlled, such as typographical errors and spelling mistakes.

Moreover, concomitantly administered drugs, age groups and indications possibly introduced confounding bias. To exclude this possible effect, a sensitivity analysis that excluded the cases where drugs other than DPP-4 inhibitors were suspected in the $\mathrm{BP}$ occurrence had been made in the study, but BP events that may be caused by unknown drugs' interactions hadn't been excluded. And the patients' other concomitant diseases or drugs or indications were limits in the FAERS report.

Additionally, the FDA did not require that a causal relationship between a product and event be proven, and reports did not always contain enough detail to properly evaluate an event. Mapping names of pharmaceutical products to an active substance is still not sufficiently resolved the issue in pharmacovigilance and epidemiology [37]. So we can use this database to generate hypotheses rather than hypotheses testing, the database can't be used to calculate the incidence of an adverse event or medication error in the United States or establish any causal relationship.

In general, further study, particularly clinical trials, is required with better data sources and research design to ensure whether Diabetes Medications have any synergistic effect on BP.

\section{Conclusion}

In conclusion, this study suggests a strong signal between bullous pemphigoid and DDP-4 inhibitors in the FAERS and the combining data from French, Japanese, WHO, Spanish and American pharmacovigilance databases. The signal was significantly higher with alogliptin than with the other DPP-4 inhibitors in the FAERS. The effect of DPP-4 inhibitors on BP did not have a statistical difference between gender in the FAERS.

The study also suggests the association between alpha-glucosidase inhibitors, meglitinides, nonsulfonylureas, sulfonylureas and BP in the FAERS. And it doesn't suggest the association between the incretin mimetics, insulin, SGLT-2 inhibitors, thiazolidinediones and BP in the FAERS.

\section{Supplementary Material}

Supplementary table 1.

http://www.medsci.org/v18p1946s1.pdf

Supplementary table 2 .

http://www.medsci.org/v18p1946s2.pdf

Supplementary table 3.

http://www.medsci.org/v18p1946s3.pdf

\section{Acknowledgements}

We acknowledge all the contributors of OpenVigil FDA and FAERS database, and the National Natural Science Foundation of China, Suzhou Science and Technology Development Project and High-tech zone health talents key talent category for the funding support.

\section{Ethics}

Since the study consisted of pharmacovigilance databases without patient contact, no approval is required.

\section{Funding}

This work was supported in part by the National Natural Science Foundation of China (81773541), funds from the Priority Academic Program Development of Jiangsu Higher Education Institutions at Soochow University, the National Key Laboratory of Radiation Medicine and Radiation Protection (GZK1201919) to ZXT. Suzhou Science and Technology Development Project (SYSD2019171) and Suzhou high-tech zone health talents key talent category (No.2019) to YGJ. The funding body did not play any roles in the design of the study and collection, analysis, and interpretation of data and in writing the manuscript.

\section{Authors' contributions}

- Study conception and design: Liting Huang, Ying Liu, Zaixiang Tang, Yiguo Jiang;

- Real data and analysis: Liting Huang;

- Drafting of the manuscript: Liting Huang, Ying Liu, Huijun Li, Weicun Huang, Ruirui Geng; 
- All authors read and approved the final manuscript.

\section{Competing Interests}

The authors have declared that no competing interest exists.

\section{References}

1. Nishie W. Update on the pathogenesis of bullous pemphigoid: an autoantibody-mediated blistering disease targeting collagen XVII. J Dermatol Sci. 2014; 73: 179-86.

2. Kalinska-Bienias A, Kowalczyk E, Jagielski P, Bienias P, Kowalewski C, Wozniak $\mathrm{K}$. The association between neurological diseases, malignancies and cardiovascular comorbidities among patients with bullous pemphigoid: Case-control study in a specialized Polish center. Adv Clin Exp Med. 2019; 28: $637-42$.

3. Hammers CM, Stanley JR. Mechanisms of Disease: Pemphigus and Bullous Pemphigoid. Annu Rev Pathol. 2016; 11: 175-97.

4. Cortes B, Khelifa E, Clivaz L, Cazzaniga S, Saurat JH, Naldi L, et al. Mortality rate in bullous pemphigoid: a retrospective monocentric cohort study. Dermatology. 2012; 225: 320-5.

5. Sagi L, Baum S, Agmon-Levin N, Sherer Y, Katz BS, Barzilai O, et al. Autoimmune bullous diseases the spectrum of infectious agent antibodies and review of the literature. Autoimmun Rev. 2011; 10: 527-35.

6. Kasperkiewicz M, Zillikens D. The Pathophysiology of Bullous Pemphigoid. Clinical Reviews in Allergy \& Immunology. 2007; 33: 67-77.

7. Sakai A, Shimomura Y, Ansai O, Saito Y, Tomii K, Tsuchida Y, et al. Linagliptin-associated bullous pemphigoid that was most likely caused by $\operatorname{IgG}$ autoantibodies against the midportion of BP180. Br J Dermatol. 2017; 176: 541-3.

8. Carnovale C, Mazhar F, Arzenton E, Moretti U, Pozzi M, Mosini G, et al. Bullous pemphigoid induced by dipeptidyl peptidase-4 (DPP-4) inhibitors: a pharmacovigilance-pharmacodynamic/pharmacokinetic assessment through an analysis of the vigibase(R). Expert Opin Drug Saf. 2019; 18: 1099-108.

9. Bene J, Moulis G, Bennani I, Auffret M, Coupe P, Babai S, et al. Bullous pemphigoid and dipeptidyl peptidase IV inhibitors: a case-noncase study in the French Pharmacovigilance Database. Br J Dermatol. 2016; 175: 296-301.

10. Stavropoulos PG, Soura E, Antoniou C. Drug-induced pemphigoid: a review of the literature. Journal of the European Academy of Dermatology and Venereology. 2014; 28: 1133-40.

11. Chuang TY, Korkij W, Soltani K, Clayman J, Cook J. INCREASED FREQUENCY OF DIABETES-MELLITUS IN PATIENTS WITH BULLOUS PEMPHIGOID - A CASE-CONTROL STUDY. Journal of the American Academy of Dermatology. 1984; 11: 1099-102.

12. Haber R, Fayad AM, Stephan F, Obeid G, Tomb R. Bullous Pemphigoid Associated With Linagliptin Treatment. JAMA Dermatol. 2016; 152: 224-6.

13. Forssmann U, Stoetzer C, Stephan M, Kruschinski C, Skripuletz T, Schade J, et al. Inhibition of CD26/Dipeptidyl Peptidase IV Enhances CCL11/EotaxinMediated Recruitment of Eosinophils In vivo. The Journal of Immunology. 2008; 181: 1120-7.

14. Skandalis K, Spirova M, Gaitanis G, Tsartsarakis A, Bassukas ID. Drug-induced bullous pemphigoid in diabetes mellitus patients receiving dipeptidyl peptidase-IV inhibitors plus metformin. J Eur Acad Dermatol Venereol. 2012; 26: 249-53.

15. Garcia-Diez I, Ivars-Lleo $M$, Lopez-Aventin D, Ishii N, Hashimoto $T$, Iranzo P et al. Bullous pemphigoid induced by dipeptidyl peptidase-4 inhibitors. Eight cases with clinical and immunological characterization. International Journal of Dermatology. 2018; 57: 810-6.

16. Aouidad I, Fite C, Marinho E, Deschamps L, Crickx B, Descamps V. A Case Report of Bullous Pemphigoid Induced by Dipeptidyl Peptidase-4 Inhibitors. Jama Dermatology. 2013; 149: 243-5.

17. Pasmatzi E, Monastirli A, Habeos J, Georgiou S, Tsambaos D. Dipeptidyl peptidase-4 inhibitors cause bullous pemphigoid in diabetic patients: report of two cases. Diabetes Care. 2011; 34: e133.

18. Bene J, Jacobsoone A, Coupe P, Auffret M, Babai S, Hillaire-Buys D, et al. Bullous pemphigoid induced by vildagliptin: a report of three cases. Fundam Clin Pharmacol. 2015; 29: 112-4.

19. Yoshiji S, Murakami T, Harashima S-i, Ko R, Kashima R, Yabe D, et al. Bullous pemphigoid associated with dipeptidyl peptidase- 4 inhibitors: A report of five cases. Journal of Diabetes Investigation. 2018; 9: 445-7.

20. Attaway A, Mersfelder TL, Vaishnav S, Baker JK. Bullous pemphigoid associated with dipeptidyl peptidase IV inhibitors. A case report and review of literature. J Dermatol Case Rep. 2014; 8: 24-8.

21. Kawaguchi Y, Shimauchi R, Nishibori N, Kawashima K, Oshitani S, Fujiya A, et al. Dipeptidyl peptidase-4 inhibitors-associated bullous pemphigoid: A retrospective study of 168 pemphigoid and 9,304 diabetes mellitus patients. J Diabetes Investig. 2019; 10: 392-8.

22. Lee SG, Lee HJ, Yoon MS, Kim DH. Association of Dipeptidyl Peptidase 4 Inhibitor Use With Risk of Bullous Pemphigoid in Patients With Diabetes. JAMA Dermatology. 2019; 155.
23. Kridin K, Cohen AD. Dipeptidyl-peptidase IV inhibitor-associated bullous pemphigoid: A systematic review and meta-analysis. J Am Acad Dermatol. 2018.

24. Balakin KV. Data Mining in Pharmacovigilance. Pharmaceutical Data Mining: https://onlinelibrary.wiley.com/doi/abs/10.1002/9780470567623.ch12; 2009. p. 339-77.

25. Hauben $M$, Reich L. Potential utility of data-mining algorithms for early detection of potentially fatal/disabling adverse drug reactions: a retrospective evaluation. J Clin Pharmacol. 2005; 45: 378-84.

26. Molina-Guarneros JA, Sainz-Gil M, Sanz-Fadrique R, Garcia P, RodriguezJimenez P, Navarro-Garcia E, et al. Bullous pemphigoid associated with the use of dipeptidil peptidase- 4 inhibitors: analysis from studies based on pharmacovigilance databases. Int J Clin Pharm. 2020; 42: 713-20.

27. Arai M, Shirakawa J, Konishi H, Sagawa N, Terauchi Y. Bullous Pemphigoid and Dipeptidyl Peptidase 4 Inhibitors: A Disproportionality Analysis Based on the Japanese Adverse Drug Event Report Database. Diabetes Care. 2018; 41: e130-e2.

28. Garcia M, Aranburu MA, Palacios-Zabalza I, Lertxundi U, Aguirre C. Dipeptidyl peptidase-IV inhibitors induced bullous pemphigoid: a case report and analysis of cases reported in the European pharmacovigilance database. J Clin Pharm Ther. 2016; 41: 368-70

29. Boehm R, Hoecker J, Cascorbi I, Herdegen T. OpenVigil-free eyeballs on AERS pharmacovigilance data. Nature Biotechnology. 2012; 30: 137-8.

30. Bohm R, von Hehn L, Herdegen T, Klein HJ, Bruhn O, Petri H, et al. OpenVigil FDA - Inspection of U.S. American Adverse Drug Events Pharmacovigilance Data and Novel Clinical Applications. PLoS One. 2016; 11: e0157753.

31. Poluzzi E, Raschi E, Piccinni C, De F. Data Mining Techniques in Pharmacovigilance: Analysis of the Publicly Accessible FDA Adverse Event Reporting System (AERS). Data Mining Applications in Engineering and Medicine; 2012. p. 273-5.

32. Evans SJ, Waller PC, Davis S. Use of proportional reporting ratios (PRRs) for signal generation from spontaneous adverse drug reaction reports. Pharmacoepidemiol Drug Saf. 2001; 10: 483-6.

33. Benzaquen M, Borradori L, Berbis P, Cazzaniga S, Valero R, Richard MA, et al Dipeptidyl peptidase IV inhibitors, a risk factor for bullous pemphigoid: Retrospective multicenter case-control study from France and Switzerland. J Am Acad Dermatol. 2018; 78: 1090-6.

34. Varpuluoma O, Forsti AK, Jokelainen J, Turpeinen M, Timonen M, Huilaja L, et al. Vildagliptin Significantly Increases the Risk of Bullous Pemphigoid: A Finnish Nationwide Registry Study. J Invest Dermatol. 2018; 138: 1659-61.

35. Gouverneur A, Lair A, Arnaud M, Bégaud B, Raschi E, Pariente A, et al. DPP-4 inhibitors and venous thromboembolism: an analysis of the WHO spontaneous reporting database. The Lancet Diabetes \& Endocrinology. 2020; 8: 365-7.

36. Kridin K, Bergman R. Association of Bullous Pemphigoid With DipeptidylPeptidase 4 Inhibitors in Patients With Diabetes. JAMA Dermatology. 2018; 154

37. https://open.fda.gov/apis/openfda-fields/. 